\title{
Stability Results for Cellular Neural Networks with Delays
}

\author{
István Győri and Ferenc Hartung \\ Department of Mathematics and Computing \\ University of Veszprém \\ H-8201 Veszprém, P.O.Box 158, Hungary \\ gyori@almos.vein.hu and hartung@szt.vein.hu
}

This paper is dedicated to Prof. László Hatvani on the occasion of his 60th birthday.

\begin{abstract}
In this paper we give a sufficient condition to imply global asymptotic stability of a delayed cellular neural network of the form

$$
\dot{x}_{i}(t)=-d_{i} x_{i}(t)+\sum_{j=1}^{n} a_{i j} f\left(x_{j}(t)\right)+\sum_{j=1}^{n} b_{i j} f\left(x_{j}\left(t-\tau_{i j}\right)\right)+u_{i}, \quad t \geq 0, \quad i=1, \ldots, n,
$$
\end{abstract}

where $f(t)=\frac{1}{2}(|t+1|-|t-1|)$. In order to prove this stability result we need a sufficient condition which guarantees that the trivial solution of the linear delay system

$$
\dot{z}_{i}(t)=\sum_{j=1}^{n} a_{i j} z_{j}(t)+\sum_{j=1}^{n} b_{i j} z_{j}\left(t-\tau_{i j}\right), \quad t \geq 0, \quad i=1, \ldots, n
$$

is asymptotically stable independently of the delays $\tau_{i j}$.

keywords: delayed cellular neural networks, global asymptotic stability, M-matrix

\section{Introduction}

The notion of cellular neural networks (CNNs) was introduced by Chua and Yang ([5]), and since then, CNN models have been used in many engineering applications, e.g., in signal processing and especially in static image treatment [6]. As a generalization of CNNs, cellular neural networks with delays (DCNNs) were introduced by Roska and Chua [14].

In this paper we study the asymptotic stability of the DCNN model described by the system of nonlinear delay differential equations

$$
\dot{x}_{i}(t)=-d_{i} x_{i}(t)+\sum_{j=1}^{n} a_{i j} f\left(x_{j}(t)\right)+\sum_{j=1}^{n} b_{i j} f\left(x_{j}\left(t-\tau_{i j}\right)\right)+u_{i}, \quad t \geq 0, \quad i=1, \ldots, n .
$$

This research was partially supported by Hungarian National Foundation for Scientific Research Grant No. T031935.

This paper is in final form and no version of it will be submitted for publication elsewhere.

EJQTDE, Proc. 7th Coll. QTDE, 2004 No. 13, p. 1 
Here $n$ is the number of cells; $x_{i}(t)$ denotes the potential of the $i$ th cell at time $t ; d_{i}$ represents the rate with which the $i$ th unit resets its potential to the resting state when it is isolated from other cells and inputs; $a_{i j}$ and $b_{i j}$ denote the strengths of the $j$ th unit on the $i$ th unit at time $t$ and $t-\tau_{i j}$, respectively; $\tau_{i j}$ corresponds to transmission delay between the $i$ th and $j$ th cells; $f$ denotes an output function; $u_{i}$ is an external input to the $i$ th cell.

The stability of (1.1) and more general classes of DCNNs has been intensively studied, see, e.g., [2]-[4], [11]-[13], [15]-[18], and the references therein. We will assume throughout this paper that the output function $f: \mathbb{R} \rightarrow \mathbb{R}$ is defined by

$$
f(t)=\frac{1}{2}(|t+1|-|t-1|)= \begin{cases}1, & t>1 \\ t, & -1 \leq t \leq 1, \\ -1, & t<-1\end{cases}
$$

This function is widely used in CNN and DCNN models.

In a recent paper Mohamad and Gopalsamy ([13]) have shown using fixed point method that if $f$ is defined by (1.2) and

$$
d_{i}>\sum_{j=1}^{n}\left(\left|a_{i j}\right|+\left|b_{i j}\right|\right), \quad i=1,2, \ldots, n,
$$

then (1.1) has a unique fixed point which is globally exponentially stable. In our Theorem 4 (see below) we show that the weaker assumption

$$
d_{i}-a_{i i}>\sum_{\substack{j=1, j \neq i}}^{n}\left|a_{i j}\right|+\sum_{j=1}^{n}\left|b_{i j}\right|, \quad i=1,2, \ldots, n,
$$

together with another condition (see (3.11) below) implies the global asymptotic stability of the unique equilibrium of (1.1). We also conjecture (see Conjecture 1 below) that assumption (3.11) can be omitted, (1.4) itself, or even a weaker condition implies the global asymptotic stability of the equilibrium.

We remark that condition (1.4) is equivalent to saying that the matrix $K=\left(k_{i j}\right)$ with elements

$$
k_{i j}= \begin{cases}d_{i}-a_{i i}-\left|b_{i i}\right|, & \text { if } i=j, \\ -\left|a_{i j}\right|-\left|b_{i j}\right| & \text { otherwise }\end{cases}
$$

is diagonally dominant and it has positive diagonal elements. We recall that an $n \times n$ matrix $K=\left(k_{i j}\right)$ is (row) diagonally dominant, if

$$
\left|k_{i i}\right|>\sum_{\substack{j=1, j \neq i}}^{n}\left|k_{i j}\right|, \quad i=1, \ldots, n .
$$

Our condition (1.4) is similar to that given by Takahashi in [15], where it was shown that if $d_{1}=d_{2}=\cdots=d_{n}=1$ and the $n \times n$ matrix $W=\left(w_{i j}\right)$ with elements

$$
w_{i j}= \begin{cases}a_{i i}-1-\left|b_{i i}\right|, & \text { if } i=j, \\ -\left|a_{i j}\right|-\left|b_{i j}\right| & \text { otherwise }\end{cases}
$$

EJQTDE, Proc. 7th Coll. QTDE, 2004 No. 13, p. 2 
is a nonsingular M-matrix (see definition below), then every solution of (1.1) tends to a constant equilibrium, i.e., the system is completely stable. Clearly, condition (1.4) implies that $d_{i}-a_{i i}>\left|b_{i i}\right|$, so in this case $W$ can not be an M-matrix. Similarly, if $W$ is an M-matrix, then (1.4) can not hold, therefore the two conditions cover disjoint cases. We comment that despite the similarities of the two conditions, the proof of our result requires a different technique than that used in [15]. Our results were motivated by the monotone technique we used in [9], where we studied the scalar version of (1.1) with $f$ defined by (1.2), and showed that the scalar version of (1.4) implies the global asymptotic stability of the unique equilibrium.

In Section 2 we give a sufficient condition which implies asymptotic stability of a linear delay system for all delays. Such stability is called absolute stability in the engineering literature. We extend a known result [3] for the case we use in Section 3 to prove our stability results for (1.1). In Section 4 we give an example to illustrate the main result and we formulate a conjecture to generalize the result.

First we introduce some notations. Let $\mathbb{R}_{+}$be the set of positive real numbers. We use the relation $\mathbf{x} \leq \mathbf{y}\left(\mathbf{x}<\mathbf{y}\right.$, respectively) for vectors $\mathbf{x}, \mathbf{y} \in \mathbb{R}^{n}$, if $x_{i} \leq y_{i}\left(x_{i}<y_{i}\right.$, respectively $)$ for all $i=1, \ldots, n$, where $\mathbf{x}=\left(x_{1}, \ldots, x_{n}\right)^{T}$ and $\mathbf{y}=\left(y_{1}, \ldots, y_{n}\right)^{T}$. We introduce the vectors $\mathbf{0}=(0,0, \ldots, 0)^{T} \in \mathbb{R}^{n}$ and $\mathbf{1}=(1,1, \ldots, 1)^{T} \in \mathbb{R}^{n}$.

For an $n \times n$ matrix $B$ the symbol $|B|$ denotes the corresponding $n \times n$ matrix with $i j$ th element $\left|b_{i j}\right|$. Similarly, $|\mathbf{u}|=\left(\left|x_{1}\right|, \ldots,\left|x_{n}\right|\right)^{T}$.

We say that an $n \times n$ matrix $K$ is an M-matrix, if all of its diagonal elements are nonnegative, and its off-diagonal elements are nonpositive, and all of its principal minors are nonnegative (see, e.g., [1], [3] or [7]). It is known (see, e.g., [1]) that if $K$ is a nonsingular M-matrix, then $\mathbf{x} \leq \mathbf{y}$ implies $K^{-1} \mathbf{x} \leq K^{-1} \mathbf{y}$.

Remark 1 Let $K$ be a matrix such that the diagonal elements of $K$ are all positive and the off-diagonal elements are all nonpositive. Then it is known (see, e.g., Theorem 2.3 in [1]) that if $K$ is a diagonally dominant, then it is a nonsingular M-matrix, as well. Moreover, $K$ is a nonsingular M-matrix, if and only if, there exists a positive diagonal matrix $D$ such that $K D$ is a diagonally dominant matrix. We note that there are 50 conditions listed in [1] which are all equivalent to that a matrix is a nonsingular M-matrix.

\section{Absolute Stability of a Linear System}

Consider the autonomous linear delay system

$$
\dot{z}_{i}(t)=\sum_{j=1}^{n} a_{i j} z_{j}(t)+\sum_{j=1}^{n} b_{i j} z_{j}\left(t-\tau_{i j}\right), \quad t \geq 0, \quad i=1, \ldots, n,
$$

where $\tau_{i j} \geq 0$ for $i, j=1, \ldots, n$.

We put the coefficients to the $n \times n$ matrices $A=\left(a_{i j}\right)$ and $B=\left(b_{i j}\right)$. For the matrix $A$ we associate the $n \times n$ diagonal matrix $A_{0}=\operatorname{diag}\left(a_{11}, a_{22}, \ldots, a_{n n}\right)$, i.e., the diagonal part of 
$A$, and let $A_{1}=A-A_{0}$ be the off-diagonal part of $A$. Then with this notation, which we use throughout this paper, we can rewrite $A$ as $A=A_{0}+A_{1}$. Similarly, let $B_{0}$ be the diagonal part of $B$, and denote $B_{1}=B-B_{0}$.

In the case when $A_{1}=0$ and $B_{0}=0$ the necessary and sufficient condition for the stability and asymptotic stability of (2.1) for all selection of the delays $\tau_{i j}$ was established in [10]. Following the methods of [10] this result was extended in [3] for the special case when only $A_{1}=0$, i.e., $A$ is a diagonal matrix in (2.1), and $B$ is an arbitrary matrix.

Theorem 1 (see Theorem 2.6 in [3]) Suppose $A=A_{0}$. Then the trivial solution of (2.1) is asymptotically stable for all delays $\tau_{i j} \geq 0$, if and only if $-A-|B|$ is an $M$-matrix and $A+B$ is a nonsingular matrix.

Note that in the case when $B$ is a nonnegative matrix, this result follows from a more general theorem in [7], where such result was proved for quasilinear delay differential equations. In the case when $B$ is a nonnegative matrix, Theorem 1 also follows from an other generalization of it given in [8], where it was shown that if $\tau_{k} \geq 0,(k=1, \ldots, p), D_{k} \geq 0$ are diagonal matrices for $k=1, \ldots, p$ such that $\sum_{k=1}^{p} D_{k}$ is invertible, $B_{\ell}$ are nonnegative $n \times n$ matrices for $\ell=1, \ldots, r$, and equation

$$
\dot{\mathbf{u}}(t)=-\sum_{k=1}^{p} D_{k} \mathbf{u}\left(t-\tau_{k}\right)
$$

has a positive fundamental solution, then the trivial solution of

$$
\dot{\mathbf{x}}(t)=-\sum_{k=1}^{p} D_{k} \mathbf{x}\left(t-\tau_{k}\right)+\sum_{\ell=1}^{r} B_{\ell} \mathbf{x}\left(t-\sigma_{\ell}\right)
$$

is asymptotically stable for all $\sigma_{1}, \ldots, \sigma_{\ell} \geq 0$, if and only if

$$
\sum_{k=1}^{p} D_{k}-\sum_{\ell=1}^{r} B_{\ell}
$$

is a nonsingular M-matrix.

We extend the sufficient part of Theorem 1 for the case which we will need later. We assume $A \neq A_{0}$, i.e., there are nonzero off-diagonal parts of $A$. The proof follows that of Theorem 1 (see [3]).

Theorem 2 Suppose $-A_{0}-\left|A_{1}\right|-|B|$ is a nonsingular M-matrix. Then the trivial solution of (2.1) is asymptotically stable for all delays $\tau_{i j} \geq 0$. 
Proof Finding the solution of $(2.1)$ in the form $e^{\lambda t} \mathbf{v}(\mathbf{v} \neq 0)$ leads to the characteristic equation

$$
\operatorname{det}\left(\begin{array}{cccc}
a_{11}+b_{11} e^{-\lambda \tau_{11}}-\lambda & a_{12}+b_{12} e^{-\lambda \tau_{12}} & \cdots & a_{1 n}+b_{1 n} e^{-\lambda \tau_{1 n}} \\
a_{21}+b_{21} e^{-\lambda \tau_{21}} & a_{22}+b_{22} e^{-\lambda \tau_{22}}-\lambda & \cdots & a_{2 n}+b_{2 n} e^{-\lambda \tau_{2 n}} \\
\vdots & \vdots & & \vdots \\
a_{n 1}+b_{n 1} e^{-\lambda \tau_{n 1}} & a_{n 2}+b_{n 2} e^{-\lambda \tau_{n 2}} & \cdots & a_{n n}+b_{n n} e^{-\lambda \tau_{n n}}-\lambda
\end{array}\right)=0
$$

of (2.1). It is known that the asymptotic stability of the trivial solution of (2.1) is equivalent to that all roots of (2.2) have negative real parts. Let $\lambda$ be a root of (2.2), then $\lambda$ is an eigenvalue of the matrix

$$
G(\lambda)=\left(\begin{array}{cccc}
a_{11}+b_{11} e^{-\lambda \tau_{11}} & a_{12}+b_{12} e^{-\lambda \tau_{12}} & \cdots & a_{1 n}+b_{1 n} e^{-\lambda \tau_{1 n}} \\
a_{21}+b_{21} e^{-\lambda \tau_{21}} & a_{22}+b_{22} e^{-\lambda \tau_{22}} & \cdots & a_{2 n}+b_{2 n} e^{-\lambda \tau_{2 n}} \\
\vdots & \vdots & & \vdots \\
a_{n 1}+b_{n 1} e^{-\lambda \tau_{n 1}} & a_{n 2}+b_{n 2} e^{-\lambda \tau_{n 2}} & \cdots & a_{n n}+b_{n n} e^{-\lambda \tau_{n n}}
\end{array}\right)
$$

Since $-A_{0}-\left|A_{1}\right|-|B|$ is a nonsingular M-matrix, it is known (see, e.g., Theorem 2.3 in [1]) there exist positive constants $\gamma_{1}, \ldots, \gamma_{n}>0$ such that

$$
\left(-a_{i i}-\left|b_{i i}\right|\right) \gamma_{i}>\sum_{\substack{j=1, j \neq i}}^{n}\left(\left|a_{i j}\right|+\left|b_{i j}\right|\right) \gamma_{j}, \quad i=1, \ldots, n .
$$

Let $\Gamma=\operatorname{diag}\left(\gamma_{1}, \ldots, \gamma_{n}\right)$. Then $\Gamma$ is nonsingular, therefore $\lambda$ is an eigenvalue of the matrix $\Gamma^{-1} G(\lambda) \Gamma$, as well. Therefore an application of Gersgorin's theorem for the matrix $\Gamma^{-1} G(\lambda) \Gamma$ yields

$$
\left|\lambda-a_{i i}-b_{i i} e^{-\lambda \tau_{i i}}\right| \leq \sum_{\substack{j=1, j \neq i}}^{n} \gamma_{i}^{-1}\left(\left|a_{i j}\right|+\left|b_{i j}\right|\left|e^{-\lambda \tau_{i j}}\right|\right) \gamma_{j}
$$

for some $i$. Therefore for this fixed $i$

$$
\operatorname{Re}(\lambda) \leq \operatorname{Re}\left(a_{i i}+b_{i i} e^{-\lambda \tau_{i i}}\right)+\sum_{\substack{j=1, j \neq i}}^{n} \gamma_{i}^{-1}\left(\left|a_{i j}\right|+\left|b_{i j}\right| e^{-(\operatorname{Re} \lambda) \tau_{i j}}\right) \gamma_{j} .
$$

Suppose $\operatorname{Re}(\lambda) \geq 0$. Then (2.3) yields

$$
\operatorname{Re}(\lambda) \gamma_{i} \leq\left(a_{i i}+\left|b_{i i}\right|\right) \gamma_{i}+\sum_{\substack{j=1, j \neq i}}^{n}\left(\left|a_{i j}\right|+\left|b_{i j}\right|\right) \gamma_{j}<0
$$

which contradicts to the assumption, therefore $\operatorname{Re}(\lambda)<0$ for all solutions of $(2.2)$.

The proof implies immediately the next technical result.

EJQTDE, Proc. 7th Coll. QTDE, 2004 No. 13, p. 5 
Corollary 3 If $-A_{0}-\left|A_{1}\right|-|B|$ is a nonsingular $M$-matrix, then $A+B$ is nonsingular, as well.

Proof Let $A$ and $B$ satisfy the assumption, pick any $\tau_{i j} \geq 0(i, j=1, \ldots, n)$, and consider the corresponding system (2.1). The proof of Theorem 2 shows that $\mathbf{v}$ is a nonzero constant solution of system (2.1) if and only if $\lambda=0$ is a solution of (2.2). But under this assumption all solutions of $(2.2)$ satisfy $\operatorname{Re}(\lambda)<0$, therefore the only constant solution of $(2.1)$ is the zero solution. On the other hand, the constant $\mathbf{v}$ solutions of $(2.1)$ satisfy $(A+B) \mathbf{v}=\mathbf{0}$, hence $A+B$ is nonsingular.

\section{Stability of a Delayed Neural Network System}

Suppose $n$ is a fixed positive integer,

$$
d_{i}>0, \tau_{i j} \geq 0, \quad a_{i j}, b_{i j}, u_{i} \in \mathbb{R}(i, j=1, \ldots, n), \quad \text { and } \quad f(t)=\frac{1}{2}(|t+1|-|t-1|) .
$$

We introduce the notations $D=\operatorname{diag}\left(d_{1}, \ldots, d_{n}\right), A=\left(a_{i j}\right), B=\left(b_{i j}\right), \mathbf{u}=\left(u_{1}, \ldots, u_{n}\right)^{T}$. As in the previous section, we use the notation $A=A_{0}+A_{1}$, where $A_{0}$ is the diagonal part, $A_{1}$ is the off-diagonal part of $A$.

Consider the DCNN model equations

$$
\dot{x}_{i}(t)=-d_{i} x_{i}(t)+\sum_{j=1}^{n} a_{i j} f\left(x_{j}(t)\right)+\sum_{j=1}^{n} b_{i j} f\left(x_{j}\left(t-\tau_{i j}\right)\right)+u_{i}, \quad t \geq 0, \quad i=1, \ldots, n
$$

with the initial conditions

$$
x_{i}(t)=\varphi_{i}(t), \quad t \in[-r, 0], \quad i=1, \ldots, n,
$$

where $r=\max \left\{\tau_{i j}: i, j=1, \ldots, n\right\}$.

To (3.2) we associate an auxiliary system. For a given $\mathbf{c}>\mathbf{0}$ and $\psi_{i}:[-r, 0] \rightarrow \mathbb{R}_{+}$ $(i=1, \ldots, n)$ consider the system

$\dot{y}_{i}(t)=-d_{i} y_{i}(t)+a_{i i} f\left(y_{i}(t)\right)+\sum_{\substack{j=1, j \neq i}}^{n}\left|a_{i j}\right| f\left(y_{j}(t)\right)+\sum_{j=1}^{n}\left|b_{i j}\right| f\left(y_{j}\left(t-\tau_{i j}\right)\right)+c_{i}, \quad t \geq 0, i=1, \ldots, n$

associated to (3.2), and the initial condition

$$
y_{i}(t)=\psi_{i}(t) \quad t \in[-r, 0], \quad i=1, \ldots, n .
$$

EJQTDE, Proc. 7th Coll. QTDE, 2004 No. 13, p. 6 
Lemma 1 Suppose (3.1). Let $\psi_{i}:[-r, 0] \rightarrow \mathbb{R}_{+}(i=1, \ldots, n), \mathbf{c}>\mathbf{0}$, and let $y_{1}, \ldots, y_{n}$ be the corresponding solution of (3.4)-(3.5). Then there exists $M>0$ such that

$$
0<y_{i}(t)<M, \quad t \geq 0, \quad i=1, \ldots, n .
$$

Proof Since $y_{i}(0)>0$ and $y_{i}$ is continuous on $[0, \infty)$ for all $i=1, \ldots, n, y_{i}(t)>0$ for small enough $t \geq 0$. Suppose there exists $i$ and $T>0$ such that

$$
y_{j}(t)>0 \quad \text { for } t \in[-r, T), j=1, \ldots, n, \quad \text { and } \quad y_{i}(T)=0 .
$$

Then $\dot{y}_{i}(T-) \leq 0$. On the other hand, (3.4) implies

$$
\dot{y}_{i}(T)=\sum_{\substack{j=1 \\ j \neq i}}^{n}\left|a_{i j}\right| f\left(y_{j}(T)\right)+\sum_{j=1}^{n}\left|b_{i j}\right| f\left(y_{j}\left(T-\tau_{i j}\right)\right)+c_{i}>0,
$$

which is a contradiction. Therefore $y_{i}(t)>0$ for all $t>0$ and $i=1, \ldots, n$.

Fix $i$. To prove that $y_{i}$ is bounded from above, assume that $\lim \sup _{t \rightarrow \infty} y_{i}(t)=\infty$. Then there exists a monotone increasing sequence $t_{n}$ such that

$$
\lim _{n \rightarrow \infty} t_{n}=\infty, \quad \lim _{n \rightarrow \infty} y_{i}\left(t_{n}\right)=\infty, \quad \text { and } \quad y_{i}\left(t_{n}\right)=\max \left\{y_{i}(t): t \in\left[-r, t_{n}\right]\right\} .
$$

Then $\dot{y}_{i}\left(t_{n}-\right) \geq 0$, which contradicts to the relations

$$
\begin{aligned}
\dot{y}_{i}\left(t_{n}\right) & =-d_{i} y_{i}\left(t_{n}\right)+a_{i i} f\left(y_{i}\left(t_{n}\right)\right)+\sum_{\substack{j=1 \\
j \neq i}}^{n}\left|a_{i j}\right| f\left(y_{j}(T)\right)+\sum_{j=1}^{n}\left|b_{i j}\right| f\left(y_{j}\left(t_{n}-\tau_{i}\right)\right)+c_{i} \\
& \leq-d_{i} y_{i}\left(t_{n}\right)+\sum_{j=1}^{n}\left|a_{i j}\right|+\sum_{j=1}^{n}\left|b_{i j}\right|+c_{i} \\
& <0
\end{aligned}
$$

for large enough $n$.

Remark 2 It is easy to check that the matrix $D-A_{0}-\left|A_{1}\right|-|B|$ is a diagonally dominant matrix with positive diagonal elements, if and only if

$$
\mathbf{0}<\left(D-A_{0}-\left|A_{1}\right|-|B|\right) \mathbf{1} .
$$

Lemma 3 Assume (3.1), $D-A_{0}-\left|A_{1}\right|-|B|$ is a diagonally dominant matrix, and

$$
\mathbf{0}<\mathbf{c}<\left(D-A_{0}-\left|A_{1}\right|-|B|\right) \mathbf{1} .
$$

Let $\psi_{i}:[-r, 0] \rightarrow \mathbb{R}_{+}(i=1, \ldots, n)$, and let $\mathbf{y}(t)=\left(y_{1}(t), \ldots, y_{n}(t)\right)^{T}$ be the corresponding solution of (3.4)-(3.5). Then

$$
\lim _{t \rightarrow \infty} \mathbf{y}(t)=\left(D-A_{0}-\left|A_{1}\right|-|B|\right)^{-1} \mathbf{c}<\mathbf{1} .
$$

EJQTDE, Proc. 7th Coll. QTDE, 2004 No. 13, p. 7 
Proof It follows from Lemma 1 that

$$
M_{i}=\limsup _{t \rightarrow \infty} y_{i}(t) \quad m_{i}=\liminf _{t \rightarrow \infty} y_{i}(t)
$$

are finite and $m_{i} \geq 0$. For a fixed $i$ there exists a sequence $t_{n}$ such that

$$
t_{n} \rightarrow \infty \quad \text { as } n \rightarrow \infty, \quad \dot{y}_{i}\left(t_{n}\right) \geq 0, n=1,2 \ldots, \quad \text { and } \quad \lim _{n \rightarrow \infty} y_{i}\left(t_{n}\right)=M_{i} .
$$

We may also assume that

$$
\lim _{n \rightarrow \infty} y_{j}\left(t_{n}\right)=m_{j}^{*} \quad \text { and } \quad \lim _{n \rightarrow \infty} y_{j}\left(t_{n}-\tau_{i j}\right)=m_{i j}^{* *}
$$

for all $j=1, \ldots, n$ for some $m_{j}^{*}, m_{i j}^{* *} \in\left[m_{j}, M_{j}\right]$, since otherwise we can select a subsequence of $t_{n}$ with this property. Then

$$
\begin{aligned}
0 & \leq \lim _{n \rightarrow \infty} \dot{y}_{i}\left(t_{n}\right) \\
& =\lim _{n \rightarrow \infty}\left(-d_{i} y_{i}\left(t_{n}\right)+a_{i i} f\left(y_{i}\left(t_{n}\right)\right)+\sum_{\substack{j=1, j \neq i}}^{n}\left|a_{i j}\right| f\left(y_{j}\left(t_{n}\right)\right)+\sum_{j=1}^{n}\left|b_{i j}\right| f\left(y_{i}\left(t_{n}-\tau_{i j}\right)\right)+c_{i}\right) \\
& =-d_{i} M_{i}+a_{i i} f\left(M_{i}\right)+\sum_{\substack{j=1, j \neq i}}^{n}\left|a_{i j}\right| f\left(m_{j}^{*}\right)+\sum_{j=1}^{n}\left|b_{i j}\right| f\left(m_{i j}^{* *}\right)+c_{i} \\
& \leq-d_{i} M_{i}+a_{i i} f\left(M_{i}\right)+\sum_{\substack{j=1, j \neq i}}^{n}\left|a_{i j}\right| f\left(M_{j}\right)+\sum_{j=1}^{n}\left|b_{i j}\right| f\left(M_{j}\right)+c_{i} .
\end{aligned}
$$

Therefore for all $i=1, \ldots, n$

$$
\begin{aligned}
c_{i} & \geq d_{i} M_{i}-a_{i i} f\left(M_{i}\right)-\sum_{\substack{j=1, j \neq i}}^{n}\left|a_{i j}\right| f\left(M_{j}\right)-\sum_{j=1}^{n}\left|b_{i j}\right| f\left(M_{j}\right) \\
& \geq d_{i} M_{i}-a_{i i} f\left(M_{i}\right)-\sum_{\substack{j=1, j \neq i}}^{n}\left|a_{i j}\right|-\sum_{j=1}^{n}\left|b_{i j}\right| .
\end{aligned}
$$

Suppose $M_{i} \geq 1$ for some $i$. Then (3.8) implies

$$
c_{i} \geq d_{i}-a_{i i}-\sum_{\substack{j=1, j \neq i}}^{n}\left|a_{i j}\right|-\sum_{j=1}^{n}\left|b_{i j}\right|
$$

which contradicts to assumption (3.6), which yields

$$
0<c_{i}<d_{i}-a_{i i}-\sum_{\substack{j=1, j \neq i}}^{n}\left|a_{i j}\right|-\sum_{j=1}^{n}\left|b_{i j}\right| .
$$

EJQTDE, Proc. 7th Coll. QTDE, 2004 No. 13, p. 8 
Therefore $0 \leq M_{i}<1$ for all $i=1, \ldots, n$. This means there exists $t_{1}>0$ such that for $t \geq t_{1}$ (3.4) is equivalent to the linear system

$$
\dot{y}_{i}(t)=\left(-d_{i}+a_{i i}\right) y_{i}(t)+\sum_{\substack{j=1, j \neq i}}^{n}\left|a_{i j}\right| y_{j}(t)+\sum_{j=1}^{n}\left|b_{i j}\right| y_{j}\left(t-\tau_{i j}\right)+c_{i}, \quad t \geq t_{1} .
$$

Define

$$
\mathbf{e}=\left(D-A_{0}-\left|A_{1}\right|-|B|\right)^{-1} \mathbf{c} .
$$

Then $\mathbf{e}=\left(e_{1}, \ldots, e_{n}\right)^{T}$ is the unique equilibrium of the system (3.9), and it follows from (3.6) that $0 \leq e_{i} \leq M_{i}<1$, so $\mathbf{0} \leq \mathbf{e}<\mathbf{1}$. Introducing $\mathbf{z}(t)=\mathbf{y}(t)-\mathbf{e}$ we can rewrite (3.9) as

$$
\dot{z}_{i}(t)=\left(-d_{i}+a_{i i}\right) z_{i}(t)+\sum_{\substack{j=1, j \neq i}}^{n}\left|a_{i j}\right| z_{j}(t)+\sum_{j=1}^{n}\left|b_{i j}\right| z_{j}\left(t-\tau_{i j}\right), \quad t \geq t_{1} .
$$

Since $D-A_{0}-\left|A_{1}\right|-|B|$ is a nonsingular M-matrix by Remark 1, Theorem 2 yields the trivial solution of (3.10) is asymptotically stable (independently of the size of the delays), therefore (3.7) holds.

Theorem 4 Assume (3.1), $D-A_{0}-\left|A_{1}\right|-|B|$ is a diagonally dominant matrix with positive diagonal elements, and $\mathbf{u}$ is such that

$$
|\mathbf{u}|<\left(D-A_{0}-\left|A_{1}\right|-|B|\right) \mathbf{1} .
$$

Then any solution $x$ of (3.2)-(3.3) satisfies

$$
\lim _{t \rightarrow \infty} \mathbf{x}(t)=(D-A-B)^{-1} \mathbf{u}
$$

Proof Fix any initial functions $\psi_{i}:[-r, 0] \rightarrow \mathbb{R}_{+}$such that

$$
\psi_{i}(s)>\left|\varphi_{i}(s)\right|, \quad s \in[-r, 0], \quad i=1, \ldots, n,
$$

and let $\mathbf{c}>|\mathbf{u}|$ be such that $\mathbf{c}<\left(D-A_{0}-\left|A_{1}\right|-|B|\right) \mathbf{1}$. Let $\mathbf{y}$ denote the solution of the corresponding IVP (3.4)-(3.5). Since $\mathbf{y}(0)>|\mathbf{x}(0)|$, relation $|\mathbf{x}(t)|<\mathbf{y}(t)$ holds for sufficiently small $t>0$. Suppose there exists $i$ and $T>0$ such that

$$
\left|x_{j}(t)\right|<y_{j}(t), \quad t \in[-\tau, T), \quad j=1, \ldots, n, \quad \text { and } \quad\left|x_{i}(T)\right|=y_{i}(T) .
$$

It follows from Lemma 1 that $\left|x_{i}(T)\right|=y_{i}(T) \neq 0$, therefore $\frac{d}{d t}\left|x_{i}(t)\right|$ exists at $T$, and $\frac{d}{d t}\left(\left|x_{i}(t)\right|\right)_{\mid t=T}=\dot{x}_{i}(T) \operatorname{sign} x_{i}(T)$. Hence

$$
\begin{aligned}
& \frac{d}{d t}\left(\left|x_{i}(t)\right|\right)_{\mid t=T} \\
& \quad=\left(-d_{i} x_{i}(T)+\sum_{j=1}^{n} a_{i j} f\left(x_{j}(T)\right)+\sum_{j=1}^{n} b_{i j} f\left(x_{j}\left(T-\tau_{i j}\right)\right)+u_{i}\right) \operatorname{sign} x_{i}(T)
\end{aligned}
$$

EJQTDE, Proc. 7th Coll. QTDE, 2004 No. 13, p. 9 


$$
\begin{aligned}
= & -d_{i}\left|x_{i}(T)\right|+a_{i i} f\left(\left|x_{i}(T)\right|\right)+\sum_{\substack{j=1, j \neq i}}^{n} a_{i j} f\left(x_{j}(T)\right) \operatorname{sign} x_{i}(T) \\
& \quad+\sum_{j=1}^{n} b_{i j} f\left(x_{j}\left(T-\tau_{i j}\right)\right) \operatorname{sign} x_{i}(T)+u_{i} \operatorname{sign} x_{i}(T) \\
< & -d_{i}\left|x_{i}(T)\right|+a_{i i} f\left(\left|x_{i}(T)\right|\right)+\sum_{\substack{j=1, j \neq i}}^{n}\left|a_{i j}\right| f\left(\left|x_{j}(T)\right|\right)+\sum_{j=1}^{n}\left|b_{i j}\right| f\left(\left|x_{j}\left(T-\tau_{i j}\right)\right|\right)+c_{i} \\
\leq & -d_{i} y_{i}(T)+a_{i i} f\left(y_{i}(T)\right)+\sum_{\substack{j=1, j \neq i}}^{n}\left|a_{i j}\right| f\left(y_{j}(T)\right)+\sum_{j=1}^{n}\left|b_{i j}\right| f\left(y_{j}\left(T-\tau_{i j}\right)\right)+c_{i} \\
= & \dot{y}_{i}(T) .
\end{aligned}
$$

This contradicts to assumption (3.13), therefore $\left|x_{i}(t)\right|<y_{i}(t)$ holds for all $t>0$ and $i=$ $1, \ldots, n$. Moreover, Lemma 3 yields

$$
\lim _{t \rightarrow \infty} \mathbf{y}(t)=\left(D-A_{0}-\left|A_{1}\right|-|B|\right)^{-1} \mathbf{c}<\mathbf{1}
$$

holds, therefore there exists $t_{1}>0$ such that $|\mathbf{x}(t)|<\mathbf{1}$ for $t \geq t_{1}$. Then (3.2) is equivalent to

$$
\dot{x}_{i}(t)=-d_{i} x_{i}(t)+\sum_{j=1}^{n} a_{i j} x_{j}(t)+\sum_{j=1}^{n} b_{i j} x_{j}\left(t-\tau_{i j}\right)+u_{i}, \quad t \geq t_{1} .
$$

This implies (3.12) using an argument similar to that in the proof of Lemma 3.

\section{Examples}

To illustrate our results consider the two-dimensional DCNN model equations

$$
\begin{aligned}
& \dot{x}_{1}(t)=-x_{1}(t)-6 f\left(x_{1}(t)\right)+f\left(x_{2}(t)\right)-3 f\left(x_{1}(t-1)\right)+f\left(x_{2}(t-2)\right)+u_{1} \\
& \dot{x}_{2}(t)=-x_{2}(t)-f\left(x_{1}(t)\right)-3 f\left(x_{2}(t)\right)-f\left(x_{1}(t-1)\right)+f\left(x_{2}(t-2)\right)+u_{2},
\end{aligned}
$$

where $f$ is defined by (1.2). It is easy to see that

$$
D-A_{0}-\left|A_{1}\right|-|B|=\left(\begin{array}{rr}
4 & -2 \\
-2 & 3
\end{array}\right)
$$

is a diagonally dominant matrix. Therefore Theorem 4 yields that if $\left|u_{1}\right|<2$ and $\left|u_{2}\right|<1$ then the trivial solution of this system is asymptotically stable. In Figure 1 we have plotted the two components of the solutions corresponding to $u_{1}=-1$ and $u_{2}=0.5$ and to the initial functions

$$
\left(\begin{array}{l}
\varphi_{1}(t) \\
\varphi_{2}(t)
\end{array}\right)=\left(\begin{array}{c}
t+1 \\
-t
\end{array}\right), \quad\left(\begin{array}{c}
\sin 2 t \\
t^{2}-1
\end{array}\right), \quad\left(\begin{array}{c}
\cos t+1 \\
t+2
\end{array}\right) \quad \text { and } \quad\left(\begin{array}{c}
t^{3}-2 \\
-2 \cos t
\end{array}\right)
$$

respectively.

EJQTDE, Proc. 7th Coll. QTDE, 2004 No. 13, p. 10 

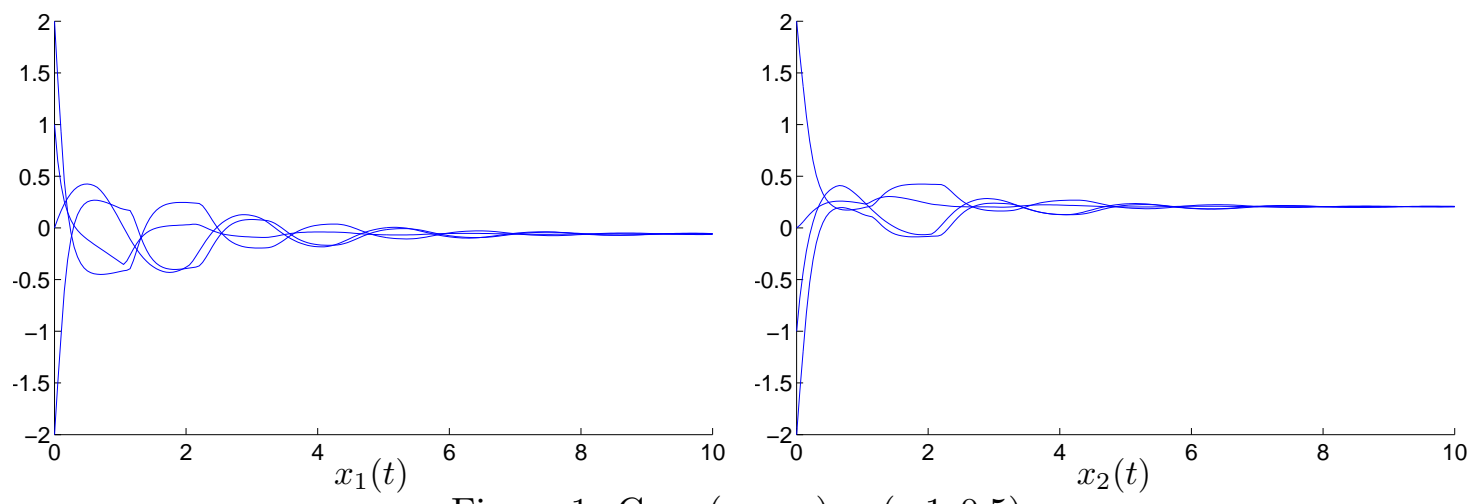

Figure 1. Case $\left(u_{1}, u_{2}\right)=(-1,0.5)$.

We can observe that all solutions tend to the unique equilibrium $(-0.058824,0.20588)^{T}$.

Note that the condition of Mohamad and Gopalsamy (1.3) is not satisfied for (4.1)-(4.2), and also the condition of Takahashi gives the matrix

$$
W=\left(\begin{array}{cc}
-7 & -2 \\
-2 & -4
\end{array}\right)
$$

which is not an M-matrix. Therefore none of this two conditions can be applied for system (4.1)-(4.2).

By checking other input values outside the region $\left|u_{1}\right|<2$ and $\left|u_{2}\right|<1$ we observed in every cases we tried all solutions tended to the unique equilibrium $\left(v_{1}, v_{2}\right)^{T}$ of the system (not necessary satisfying $\left.\left|v_{1}\right|,\left|v_{2}\right|<1\right)$. In Figure 2 we can see the graphs of solutions of (4.1)-(4.2) corresponding to $\left(u_{1}, u_{2}\right)=(3,5)$ and to the initial functions (4.3). We can observe that all solutions tend to the unique equilibrium $(0.5,2)^{T}$.
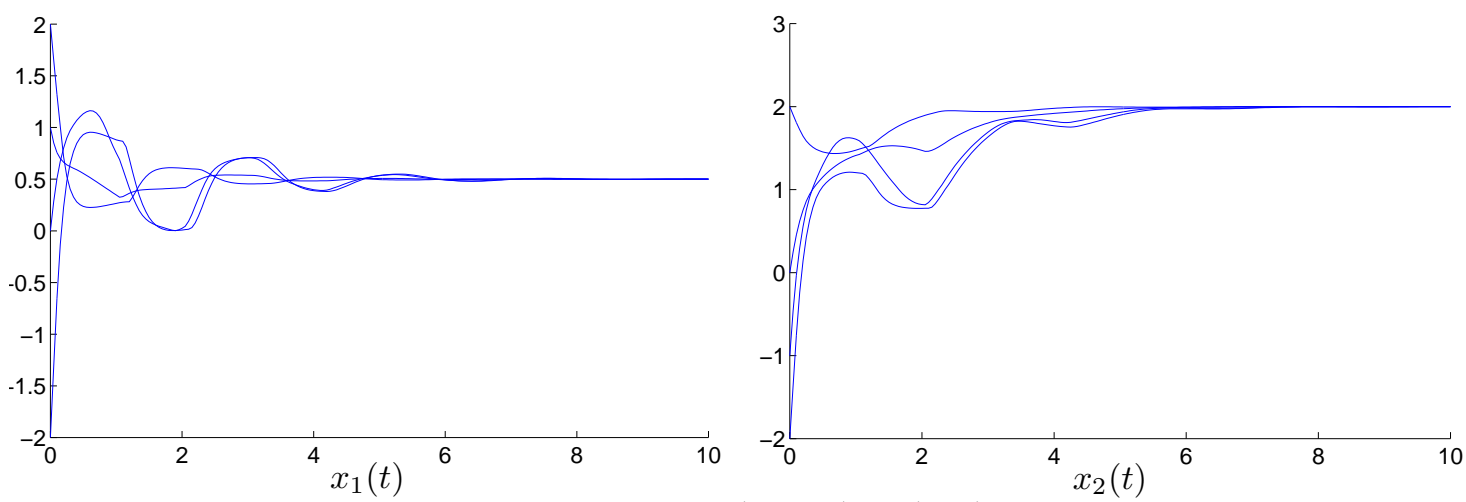

Figure 2. Case $\left(u_{1}, u_{2}\right)=(3,5)$.

Next we plotted the solutions corresponding to $\left(u_{1}, u_{2}\right)=(-8.5,-5.5)$ and to the initial functions (4.3) in Figure 3. Again, all solutions tend to the unique equilibrium $(-1.5,-1.5)^{T}$.

EJQTDE, Proc. 7th Coll. QTDE, 2004 No. 13, p. 11 

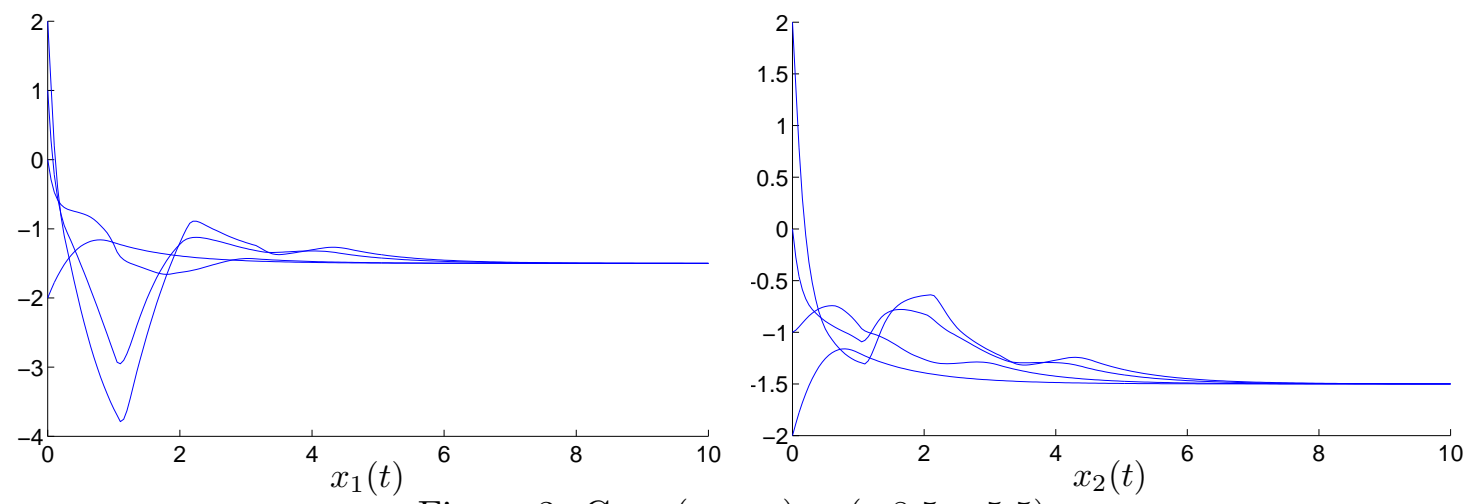

Figure 3. Case $\left(u_{1}, u_{2}\right)=(-8.5,-5.5)$.

Now change the coefficient of $f\left(x_{2}(t-2)\right)$ in (4.1) to 4, i.e., consider the system

$$
\begin{aligned}
& \dot{x}_{1}(t)=-x_{1}(t)-6 f\left(x_{1}(t)\right)+f\left(x_{2}(t)\right)-3 f\left(x_{1}(t-1)\right)+4 f\left(x_{2}(t-2)\right)+u_{1} \\
& \dot{x}_{2}(t)=-x_{2}(t)-f\left(x_{1}(t)\right)-3 f\left(x_{2}(t)\right)-f\left(x_{1}(t-1)\right)+f\left(x_{2}(t-2)\right)+u_{2} .
\end{aligned}
$$

We plotted the solutions corresponding to $\left(u_{1}, u_{2}\right)=(-6,4)$ and to the initial functions (4.3) in Figure 4. As before, all solutions tend to the unique equilibrium, which is $(-0.1,2.2)^{T}$ in this case. On the other hand,

$$
D-A_{0}-\left|A_{1}\right|-|B|=\left(\begin{array}{rr}
4 & -5 \\
-2 & 3
\end{array}\right)
$$

is no longer a diagonally dominant matrix, but it is a nonsingular M-matrix.
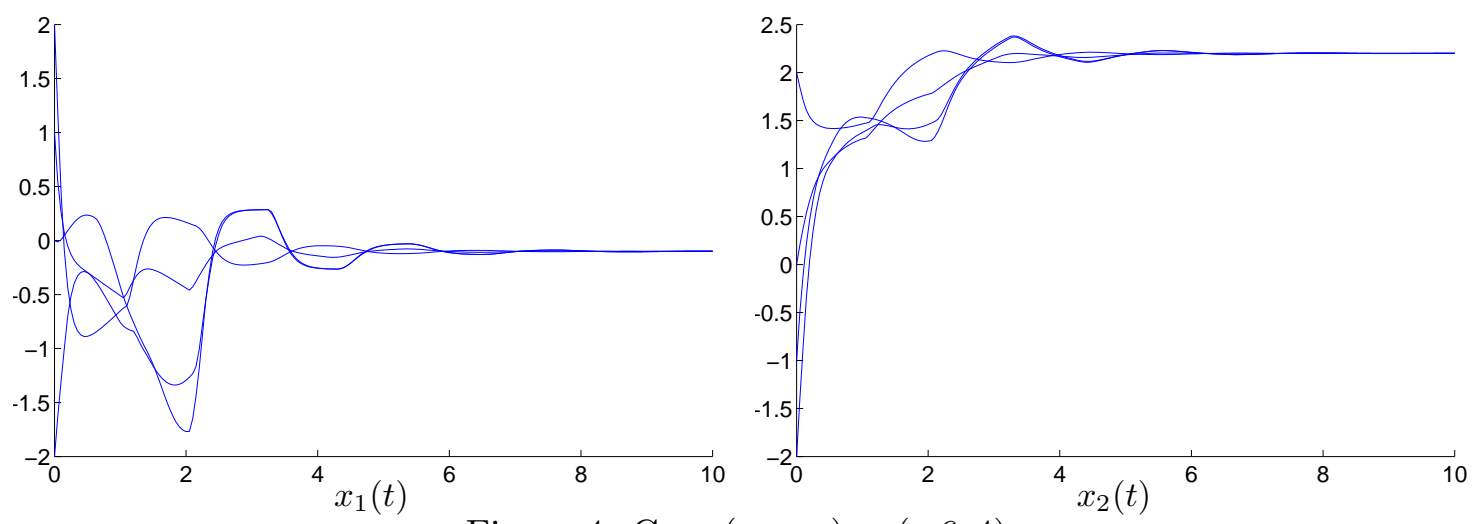

Figure 4. Case $\left(u_{1}, u_{2}\right)=(-6,4)$.

Therefore our numerical experiments on these and other systems suggest the following conjecture.

Conjecture 1 Assume (3.1) and $D-A_{0}-\left|A_{1}\right|-|B|$ is a nonsingular M-matrix. Then (3.2) has a unique equilibrium for any input vector $\mathbf{u}$, and any solution of (3.2) tends to this equilibrium.

EJQTDE, Proc. 7th Coll. QTDE, 2004 No. 13, p. 12 


\section{References}

[1] A. Berman and R. J. Plemmons, "Nonnegative Matrices in the Mathematical Sciences", Academic Press, New York, 1979.

[2] S. A. Campbell, Stability and bifurcation of a simple neural network with multiple time delays, Fields Inst. Commun. 21 (1999) 65-79.

[3] S. A. Campbell, Delay independent stability for additive neural networks, Differential Equations Dynam. Systems, 9:3-4 (2001) 115-138.

[4] J. Cao, Global exponential stability and periodic solutions of delayed cellular neural networks, J. Comput. System Sci. 60 (2000) 38-46.

[5] L. O. Chua and L. Yang, Cellular neural networks: Theory, IEEE Trans. Circuits and Systems I 35 (1988) 1257-1272.

[6] L. O. Chua, CNN: A paradigm for complexity, World Scientific, Singapore, 1998.

[7] J. Eller, Stability of quasimonotone linear autonomous functional differential equations via M-matrices, Differential Equations: Qualitative Theory, Vol I.,II. (Szeged, 1984), 251-281, B. Sz-Nagy, L. Hatvani eds., Colloquia Mathematica Societatis János Bolyai, vol. 47, North-Holland Publishing Co., Amsterdam, 1987.

[8] I. Győri, Interaction between oscillations and global asymptotic stability in delay differential equations, Differential Integral Equations 3:1 (1990) 181-200.

[9] I. Györi and F. Hartung, Stability Analysis of a Single Neuron Model with Delay, J. Comput. Appl. Math. 157:1 (2003) 73-92.

[10] J. Hofbauer and J. W.-H. So, Diagonal dominance and harmless off-diagonal delays, Proc. Amer. Math. Soc. 128 (2000) 2675-2682.

[11] M. Joy, Results concerning the absolute stability of delayed neural networks, Neural Networks 13 (2000) 613-616.

[12] X. Liao, Z. Wu and J. Yu, Stability analyses of cellular neural networks with continuous time delay, J. Comput. Appl. Math. 143 (2002) 29-47.

[13] S. Mohamad and K. Gopalsamy, Exponential stability of continuous-time and discretetime cellular neural networks with delays, Appl. Math. Comput. 135 (2003) 13-38.

[14] T. Roska and L. O. Chua, Cellular neural networks with nonlinear and delay-type template elements and non uniform grids, Internat. J. Circuit Theory Appl. 20 (1992) 469481.

[15] N. Takahashi, A new sufficient condition for complete stability of cellular neural networks with delay, IEEE Trans. Circuits Systems I. Fund. Theory Appl. 47:6 (2000) 793-799.

[16] D. Xu, H. Zhao and H. Zhu, Global dynamics of Hopfield neural networks involving variable delays, Comput. Math. Appl. 42 (2001) 39-45.

EJQTDE, Proc. 7th Coll. QTDE, 2004 No. 13, p. 13 
[17] J. Zhang, Global stability analysis in delayed cellular neural networks, Comput. Math. Appl. 45 (2003) 1707-1720.

[18] J. Zhang and X. Jin, Global stability analysis in delayed Hopfield neural network models, Neural Networks 13 (2000) 745-753.

(Received September 29, 2003)

EJQTDE, Proc. 7th Coll. QTDE, 2004 No. 13, p. 14 